\title{
PHASE TRANSITION AS AN EMERGENT PHENOMENON ANALYSED BY VIOLATION OF STRUCTURAL INVARIANT (M, BM)
}

\author{
Jiri Bila ${ }^{\bowtie}$, Ali H. Reshak, Jan Chysky \\ Department of Instrumentation and Control Engineering, Czech Technical University in Prague, Czech Republic \\ jiri.bila@fs.cvut.cz ${ }^{\bowtie}$, AliH.Reshak.AL-JAARY@fs.cvut.cz, Jan.Chysky@fs.cvut.cz
}

\begin{abstract}
When modeling complex systems, we usually encounter the following difficulties: partiality, large amounts of data and uncertainty of conclusions. The most common approach used for modeling is the physical approach, sometimes reinforced by statistical procedures. If we assume emergences in the complex system, a physical approach is not appropriate at all. Instead, we build here the approach of structural invariants. In this paper, we show that another plane can be built above the plane of physical description, which is responsible for violation of structural invariants. Main attention is concentrated (in this article) on the invariant matroid and bases of matroid $(M, B M)$ in combination with Ramsey graph theory. In addition, the article introduces a calculus that describes the emergent phenomena using two quantities - the power of the emergent phenomenon and the complexity of the structure of the considered complex system. We show the application of the method for modeling phase transition in chemistry.
\end{abstract}

Keywords: Modeling, complex systems, structural invariant, matroid, matroid bases, Ramsey theory, phase transition.

\section{Introduction}

The field of complex systems is proving to be a very needed field of research nowadays. When two years ago they were written on the topic of complex systems and emerging behavior, especially conference articles, today the papers with mentioned topics are published in very serious journals and there are referenced more than 100 papers on WoS only for the year 2020 till now. In this article, we focus attention mainly on modeling complex systems and processing emergent situations in the field associated with crystal chemistry. Emergent situations are associated in our paper with violation of so called structural invariants.

Nice examples of it we find in particle physics and we remind here works of Laughlin (reminding Goldstone's theorem in this context) [13] and also of Drapper [8] citing: "The supersymmetry break can not be done permanently by the particles of MSSM (Minimal Supersymmetric Standard Model) as they currently appear. This means that there is a new sector of the theory that is responsible for the breaking."

We present this in our article, where we build another plane above the plane of physical and crystallographic description, which describes the violation of the structural invariant (M, BM).

\section{Related Work}

In this Section we introduce only a few sources to understand our approach to the problem. Theory and applications of complex systems are, e.g., in [19, 6, 12]. For comparison of various interpretation of concepts of emergence and emergent behavior we introduce $[10,11,2,4]$. The references $[7,9,16,17]$ are introduced as a line to the fields of computational and crystalographic chemistry. Our method of analysis and the detection of emergent situations has been published many times, e.g., in $[5,1,3,4]$. Theoretical background for this method is in $[14,15,18,20]$.

\section{The Analysis and the Detection of EMSs by Structural Invariant (M, BM)}

\subsection{Matroids and Ramsey Theory of Graphs}

A matroid is usually introduced [14] as the following structure:

$$
M=\left\langle X, I N D,\left\{N_{1}, N_{2}, \ldots, N_{n}\right\}\right\rangle=\langle X, \boldsymbol{M B}\rangle,
$$

where $X$ is the ground set of elements (compartment), $I N D$ is a relation of independence, $N_{1}, N_{2}, \ldots, N_{n}$ are independent sets, and $\boldsymbol{M B}$ is a set of matroid bases.

An extension of a matroid basis by at least one element is considered (in this paper) as a violation of structural invariant (M, BM) and it is considered as an internal indicator of the appearance of an emergent situation. 


\section{Extension of matroid basis}

The extension of a basis of the matroid can be done by two ways:

m1) From the cardinality of the matroid, we calculate the minimum number of elements that must be added to the matroid to have an extending element among them. The number "min $\Delta f(R N)$ " is a minimal difference between the next Ramsey number and the current Ramsey number.

m2) We find an extending element, verify the possibility of the extension of some basis $(I N D)$ and if it is necessary we make normalization (explanation in sub section 3.2).

\section{Association with Ramsey Theory of Graphs [15]:}

Speaking about graph matroids we consider many possible graphic architecture of them - e.g. in [14]. Relations DNT and IND are considered as binary relations and they are represented by edges with connected and disconnected nodes in graphs. In the following text we work with matroids that are formed on perfect graphs and the following consequences can be used:

The bases $(\boldsymbol{M} \boldsymbol{B})$ will be constructed as perfect subgraphs in a perfect graph on $X$. (A perfect graph on $X$ has each node connected with all other nodes of $X$.)

The independent and dependent elements in a perfect graph $G_{p}=(V, E)$ are easily constructed by coloring the edges of the perfect graph by two colors, and the formalism of Ramsey numbers $-\boldsymbol{R}(\# \boldsymbol{B}, \# Y), \boldsymbol{B} \in$ $\boldsymbol{M B}$ is offered for use. We now introduce a free formulation of the relations on a perfect graph $G_{p}$ colored by two colors as a consequence of the famous Ramsey theorem:

Theorem. A perfect graph $G_{p}=(V, E)$ with $n$ nodes, where each edge belongs to class $A$ or to class $B$, contains a perfect subgraph with a nodes and edges from class $A$ or a perfect subgraph with $b$ nodes and edges from class $B$.

In most cases holds $(\# \boldsymbol{B}+\# Y) \neq n$, for $\boldsymbol{R}(\# \boldsymbol{B}, \# Y), \boldsymbol{B} \in \boldsymbol{M} \boldsymbol{B}$. The reason is that remaining nodes $(n-\# \boldsymbol{B}-\# Y)$ belong to class $\mathrm{A}$ or to class B however they do not form perfect subgraphs. Number $R(a, b)=n$ that corresponds to numbers $a$ and $b$ and is equal to number of nodes of the perfect graph $G_{p}$ is called a Ramsey number.

\subsection{The Power and Complexity of an Emergent Phenomenon}

The emergent phenomenon we describe - in our view - by two variables - the power $H_{P}$ of emergent phenomenon and the complexity $H_{C O M}$ of emergent phenomenon. Essential relations between the power $H_{P}$ and the complexity $H_{C O M}$ are expressed by two equations

$$
\begin{aligned}
& H_{P}(\boldsymbol{B}+\mathbf{1})=H_{P}(\boldsymbol{B})+(u / c) H_{C O M}(\boldsymbol{B}), \\
& H_{C O M}(\boldsymbol{B}+\mathbf{1})=H_{C O M}(\boldsymbol{B})+u H_{P}(\boldsymbol{B}),
\end{aligned}
$$

where $\boldsymbol{B} \in \boldsymbol{M} \boldsymbol{B}$ is a basis of a matroid and $\boldsymbol{B}+\mathbf{1}$ is the basis $\boldsymbol{B}$ extended by one element. Variables $H_{P}(\boldsymbol{B}), H_{P}(\boldsymbol{B}+\mathbf{1})$ and $H_{C O M}(\boldsymbol{B}), H_{C O M}(\boldsymbol{B}+\mathbf{1})$ are power and complexity of emergent phenomenon related to bases $\boldsymbol{B}$ and $\boldsymbol{B}+\mathbf{1}$. Symbol $u$ denotes the quotient of self organization of the considered complex system (represented by the compartment) and $c$ is the limit of this quotient (the best self organization). Quotient $(u / c) \in\langle 0,1\rangle$ represents "intelligence" of self organizing process that will execute the emergent phenomenon. Operating with equation (2) we obtain contribution to power released by emergent phenomenon

$$
\Delta H_{P}(\boldsymbol{B}+\mathbf{1})=(u / c) H_{C O M}(\boldsymbol{B}),
$$

where $\Delta H_{P}(\boldsymbol{B}+\mathbf{1})$ represents the changes in the system due to EMS by quantities of symptoms and $H_{C O M}(\boldsymbol{B})$ represents the complexity of the system by structure of drivers that provide $\Delta H_{P}(\boldsymbol{B}+\mathbf{1})$.

The link between $\Delta H_{P}(\boldsymbol{B}+\mathbf{1})$ and $H_{C O M}(\boldsymbol{B})$ is provided by $(u / c)$ - the "intelligence" quotient of the system. The quantity of $(u / c)$ is done by rough estimation $[4,6]$ and by soft tuning according to Appendix A.

The contribution to power of the emergent phenomenon $\Delta H_{P}(\boldsymbol{B}+\mathbf{1})$ that has an intuitive meaning in the internal level is associated in the external level of the description with the power $\Delta H_{D}(\boldsymbol{B}+\mathbf{1})$ (e.g., damage of houses by floods) measured by quantities of external variables (symptoms) $s_{i}, i=1, \ldots, n$ for emergent $\left(s_{\text {iem }}\right)$ and for nominal $\left(s_{\text {inom }}\right)$ situations:

$$
\begin{gathered}
\Delta H_{D}(\boldsymbol{B}+\mathbf{1})=\left(\sum_{i=1}^{n}\left(\omega_{i}\left(s_{\text {iem }} / s_{\text {inom }}\right)^{2}\right)\right)^{1 / 2}, \\
\Delta H_{P}(\boldsymbol{B}+\mathbf{1})=\Lambda \cdot \Delta H_{D}(\boldsymbol{B}+\mathbf{1}),
\end{gathered}
$$

where $\omega_{i}$ are quotients of importance and $\Lambda$ is a calibration constant. Quotients of importance $\omega_{i}$ are computed by Saaty method [18].

The contribution to power of emergent phenomenon $\Delta H_{D}(\boldsymbol{B}+\mathbf{1})$ results in a dimensionless real number expressed here in \% (for example, contribution for $20 \%$ is calculated as $(120 / 100)=1.2)$. Equation (3) is associated with equation (4) where $H_{C O M}(\cdot)$ is approximated in our case by number of elements of basis $\boldsymbol{B}$, i.e. $\# \boldsymbol{B}$. Function $\xi$ transforms real number $\left(\Delta H_{P}(\boldsymbol{B}+\mathbf{1}) /(u / c)\right)$ into nearest larger integer and holds:

$$
\xi\left(\Delta H_{P}(\boldsymbol{B}+\mathbf{1}) /(u / c)\right)=\# \boldsymbol{B}
$$

(For explanation: We describe a contribution $\Delta H_{D}(\boldsymbol{B}+\mathbf{1}$ of power of a possible EMS by approximating the emergent and nominal values of the selected variables in the compartment of real world. Using calibration constant $\Lambda$ we find $\Delta H_{P}(\boldsymbol{B}+\mathbf{1}$. 
Then we form a corresponding representation in the pre-geometry (matroid and matroid bases) and we compute by (7) the cardinality $\# \boldsymbol{B}$. The violation of this invariant we solve as an extension of a matroid basis. Then if necessary are computed conditions for the proposed EMS in the original compartment of real world - called here "normalization".)

We formulate now a basic rule for analyzing (detecting) an emergent situation as follows:

For case m1):

$$
\mathrm{IF}(\# E 1 \geq \min \Delta f(R N)) \Rightarrow(\mathrm{PAES}),
$$

where $E 1$ is a set that extends the matroid $\langle X 1, \boldsymbol{M} \boldsymbol{B} \mathbf{1}\rangle$ and contains at least one element $e 1$ extending basis $\boldsymbol{B 1}$. The number " $\min \Delta f(R N)$ " is the minimal difference between the next Ramsey number and the current Ramsey number, and PAES denotes the "Possible Appearance of an Emergent Situation". The number $\Delta f(R N)$ provides that at least one element could be connected with relation $I N D$ with all elements of some basis from $\boldsymbol{M} \boldsymbol{B} \mathbf{1}$. (This is the case for $\# \boldsymbol{B}$ not for $\# Y$ - in the formalism of Ramsey numbers $R(\# \boldsymbol{B}, \# Y))$.

For case m2):

$$
\operatorname{IF}\left(\exists e,\left(I N D\left(e, x_{i}\right), \forall x_{i} \in \boldsymbol{B 1}\right)\right) \Rightarrow(\mathrm{PAES}),
$$

where $e$ is an extending element, for which was verified Independence $(I N D)$ to all elements of $\boldsymbol{B} \mathbf{1}$.

\subsection{Normalization of $\Delta f(R N)$ with Regard to a Real "Volume" of Added Elements}

The following operation is actual for cases where the detecting of PAES in pre-geometry can be represented by the addition of real elements to the real compartment (e.g., drivers in an ecosystem) or the addition of some time interval to given time moment $[6]$ in the considered compartment. (We are speaking about an additive representation of drivers.)

The number $V(\boldsymbol{B})$ is different from $R N(\boldsymbol{B})$ - in general. Then is necessary to execute a normalization of $\Delta f(R N)$ to actual number of active elements $V(\boldsymbol{B})$ in the actual state of complex system. The information about number $V(\boldsymbol{B})$ is a complementary information and it does not influence the calculation according equations (4), (5), (7). The normalization procedure for the alternative "m1" (expression (8)) has the following steps:

- Let us assume that we have computed $\Delta H_{P}(\boldsymbol{B}+\mathbf{1})$ (equations (5) and (6)).

- From this number is possible to acquire $\# \boldsymbol{B}$ by equation (7).

- Considering \# $\boldsymbol{B}$ as a number of elements of a basis we compute $R N(\boldsymbol{B})$.

- This number corresponds to an actual state of the complex system and thus to a "volume" of elements in this state.
- $R N(\boldsymbol{B})$ corresponds to $V(\boldsymbol{B})$.

- From this correspondence we extract a normalization quotient $\lambda$

$$
\lambda=V(\boldsymbol{B}) / R N(\boldsymbol{B}) .
$$

- After computation $R N(\boldsymbol{B}+\mathbf{1})$ we extrapolate the quantity $V(\boldsymbol{B}+\mathbf{1})$

$$
V(\boldsymbol{B}+\mathbf{1})=\lambda R N(\boldsymbol{B}+\mathbf{1}) .
$$

- Then the value $\Delta f(R N)$ corresponds to value $\Delta V(\boldsymbol{B}, \boldsymbol{B}+\mathbf{1})$ (the number of elements that is necessary to add to $V(\boldsymbol{B}))$

$$
\Delta V(\boldsymbol{B}, \boldsymbol{B}+\mathbf{1})=\lambda \Delta f(R N)
$$

And the addition of $\Delta V(\boldsymbol{B}, \boldsymbol{B}+\mathbf{1})$ to the number of active elements of the actual state is associated with PAES.

\section{Phase Transition as an EMS Analyzed by Violation of $(\mathrm{M}, \mathrm{BM})$}

In this subsection we work with experimental and computed data acquired from [16]. The influence of phase transition on the electronic structure and the optical properties of $\mathrm{BaThO}_{3}$ was investigated by means of Density Functional Theory [16, 9]. At room temperature $\mathrm{BaThO}_{3}$ is stable in the Pbnm phase till the $547^{\circ} \mathrm{C}$, whereas it is stable in the Ibmm phase at higher temperature. The transfer from Pbnm phase to Ibmm phase by the influence of temperature we consider as an emergent situation. In Table 1 there are introduced the quantities for Pbnm phase and Ibmm phase computed by program wien $2 \mathrm{k}[7]$ :

Table 1: Values of components for phases Pbnm and Ibmm.

\begin{tabular}{|c|c|c|}
\hline Component $\left(\mathrm{Q}_{\mathrm{x}}\right)$ & $\mathrm{Q}_{\mathrm{Pbnm}}$ & $\mathrm{Q}_{\mathrm{Ibmm}}$ \\
\hline$\varepsilon_{1}^{x x}(0)$ & 3.234 & 3.273 \\
\hline$\varepsilon_{1}^{y y}(0)$ & 3.137 & 3.173 \\
\hline$\varepsilon_{1}^{z z}(0)$ & 3.116 & 3.079 \\
\hline$\delta_{\varepsilon}$ & -0.033 & -0.046 \\
\hline$\omega_{p}^{x x}$ & 8.530 & 8.966 \\
\hline$\omega_{p}^{y y}$ & 8.095 & 8.068 \\
\hline$\omega_{p}^{z z}$ & 8.449 & 7.768 \\
\hline$n^{x x}(0)$ & 1.798 & 1.809 \\
\hline$n^{y y}(0)$ & 1.771 & 1.781 \\
\hline$n^{z z}(0)$ & 1.765 & 1.754 \\
\hline$\Delta n(0)$ & -0.030 & -0.041 \\
\hline
\end{tabular}


The content of variables:

- QPbnm ...quantities of components for Pbnm phase,

- QIbmm ...quantities of components for Ibmm phase,

- $\varepsilon_{1}^{x x}(0), \varepsilon_{1}^{y y}(0), \varepsilon_{1}^{z z}(0)$ are real parts of tensor components of dielectric function in frequency 0 ,

- $\omega_{p}^{x x}, \omega_{p}^{y y}, \omega_{p}^{z z}$ are real parts of tensor components of plasmon oscillations,

- $\delta_{\varepsilon}$ is uniaxial anisotropy,

- $n^{x x}(0), n^{y y}(0), n^{z z}(0)$ are tensor components of refractive index in frequency 0 ,

- $\Delta n(0)$ represents birefringence.

As a power of emergent phenomenon is considered cumulative result of changes of the optical properties for Pbnm and Ibmm phases.

For computation $\Delta H_{D}(\boldsymbol{B}+\mathbf{1})$ we use dimensionless expression ( $\mathrm{Q}_{\mathrm{Ibmm}} \mathrm{Q}_{\mathrm{Pbnm}}$ ) for all quantities from Table 1, quotients $\omega_{i}$ of importance (equation (5)) are equal to 1 , for calibration constant $\Lambda=1$ (equation (6)) so that $\Delta H_{P}(\boldsymbol{B}+\mathbf{1})=\Delta H_{D}(\boldsymbol{B}+\mathbf{1})$.

$$
\begin{aligned}
& \Delta H_{D}(\boldsymbol{B}+\mathbf{1})=\left((3.273 / 3.234)^{2}+\right. \\
& \quad+(3.173 / 3.137)^{2}+(3.079 / 3.116)^{2}+ \\
& \quad+(-0.046 /-0.033)^{2}+(8.966 / 8.530)^{2}+ \\
& \quad+(8.068 / 8.095)^{2}+(7.768 / 8.449)^{2}+ \\
& \quad+(1.809 / 1.798)^{2}+(1.781 / 1.77 .)^{2}+ \\
& \left.\quad+(1.754 / 1.765)^{2}\right)^{1 / 2}=3.33
\end{aligned}
$$

$$
\# \boldsymbol{B}=\xi\left(\Delta H_{P}(\boldsymbol{B}+\mathbf{1}) /(u / c)\right)=9
$$

for $(\mathrm{u} / \mathrm{c})=0.37$ (after tuning operation according Appendix A.)

$$
\begin{aligned}
& \Delta f(R N)=R N(x, 10)-R N(y, 9): \\
& R N(y, 9):(115,141,153) \\
& R N(x, 10):(149,442,1171) \\
& \Delta f(R N)=\min \{(149-115),(442-141),(1171-153)\} \\
& =34 \\
& \lambda=547 / 115=4.76 \\
& \Delta T=\lambda \Delta f(R N)=161.84^{\circ} \mathrm{C}
\end{aligned}
$$

The quantity of temperature that is needed for the considered phase transfer is:

$$
547^{\circ} \mathrm{C}+161.84^{\circ} \mathrm{C}=708.84^{\circ} \mathrm{C}
$$

And it corresponds to results of experimental verification the phase transition and its consequences [16].
This introduced result should not converge on the idea that there is presented a new method of the synthesis of new chemical compounds. The result that we published in [16] was useful to support our method of representing the emergence by the expansion of the matroid base and to demonstrate emergence by the violation of another structural invariant in the lower plane - symmetry. (This is nice to see on the crystallographic groups Pbnm and Ibmm as well as on the structural grids.) The method of representation of emergence used does not go towards describing the product that emerges. It is too vague for that (PAES). Its aim is to discover the conditions under which emergences can be expected. In the article, we described only additive conditions. (PAES is conditioned by the addition, (may be connection) of a certain quantity of active scalar variable to the current state.) There are, of course, non-additive PAES conditions that are considerably more interesting.

\section{Conclusion}

The paper presents one application of our method for the processing emergent situations in complex system. While the previous published examples were oriented to emergent phenomena on macrostructure of systems (floods, traffic jams [2], onset of diseases [3]) the proposed paper describes emergent situation in the development of microstructure in crystalographic chemistry.

Acknowledgement: This research has been supported by Research Grant RVO 12000 Institutional support for the development of a research organization. This support is very gratefully acknowledged.

\section{References}

[1] Bila, J. The detection of emergent situations by structural invariants. In Proceedings of 17th International Conference on Soft Computing MENDEL 2011 (2011), R. Matousek, Ed., VUT Press, pp. 534-539.

[2] Bila, J. Modeling of complex systems by means of partial algebras. In Proc. of Interdisciplinary Symposium of Complex Systems (2014), A. Sanayei, I. Zelinka, and O. Rossler, Eds., Springer: Heidelberg, Germany, pp. 89-100.

[3] Bila, J. Emergent phenomena in complex systems and their detection. International Journal of Enhanced Research in Science Technology and Engineering 6 (2017), 40-53.

[4] Bila, J. Emergent phenomena in complex systems. Advances in Intelligent Systems and Computing 837 (2019), 262-270.

[5] Bila, J., AND NovaK, M. Detection of emergent situations in complex systems by structural invariant (MB, M). MENDEL 23 (2017), 163-170.

[6] Bila, J., Rodriguez, R., And Novak, M. Emergent phenomena in natural complex systems. MENDEL 25 (2019), 103-110. 
[7] Blaha, P., Schwarz, K., Madses, G., KvasNICKA, D., AND LuITZ, J., 2001. wien2k, An Augmented plane wave - Local Orbitals. Program for Calculating Crystal Properties.

[8] Draper, P., Meade, P., Reece, M., And SHIH, D. Implication of a 125 gev higgs for the mssm and low-scale susy breaking. Physical Review D 85 (2012), Article ID 095007.

[9] Hohenberg, P., And Kohn, W. Inhomogeneous electron gas. Physical Review 136 (1964), Article ID B864.

[10] Holland, T. Foundations for the modeling and simulation of emergent behavior systems. In Engineering Emergence: Modeling and Simulation Approach (2018), L. Rainey and M. Jamshidi, Eds., CRS Press /Taylor and Francis: Boca Raton, USA, pp. 217-258.

[11] Johnson, J., And PAdilla, J. Ontology of emergence. In Engineering Emergence: Modeling and Simulation Approach (2018), L. Rainey and M. Jamshidi, Eds., CRS Press / Taylor and Francis: Boca Raton, USA, pp. 185-198.

[12] Kotyrba, M., Volna, E., And Bujok, P. Unconventional modeling of complex system via cellular automata and differential evolution. Swarm and Evolutionary Computation 25 (2015), 52-62.

[13] Laughlin, R. A Different Universe (Reinventing Physics from the Bottom Down). Basic Books, New York, USA, 2006.

[14] Oxley, J. Matroid Theory. Oxford Science Publications, Oxford, UK, 2001.

[15] Ramsey, F. On a problem of formal logic. Proceedings of the London Mathematical Society 30 (1930), 264-286.

[16] Reshak, A., Alahmed, Z., And Bila, J. Phase transition in BaThO3 from Pbnm to Ibmm turn the fundamentals energy band gap from indirect to direct. Journal of Alloys and Compounds 771 (2019), 607-613.

[17] Reshak, A., Alahmed, Z., Bila, J., AND ET AL. Exploration of the electronic structure of monoclinic alpha-Eu2(MoO4)(3): DFT-based study and X-ray photoelectron spectroscopy. The Journal of Physical Chemistry C 120 (2016), 10559-10568.

[18] SaAty, T. Exploring the inference between hierarchies, multiple objectives and fuzzy sets. Fuzzy Sets and Systems 1 (1978), 57-68.

[19] Siegenfeld, A., And Bar-Yam, Y. An introduction to complex systems science and its applications. Complexity (2020), Article ID 6105872.

[20] Weinstein, W. Ramsey Number. A Wolfram Web Resource: https://mathworld.wolfram.com/ RamseyNumber.html.

\section{Appendix A.}

The method of soft tuning of $(u / c)$ is as follows: Considering the complexity of the compartment of the complex system given by the number of a matroid basis $H_{C O M}(\boldsymbol{B})=\# \boldsymbol{B}$ - the procedure for calculating and determining the coefficient $(u / c)$ is based on the following limitations and conditions:

(a) $(u / c) \in\langle 0,1\rangle$.

(b) $\Delta H_{P}(\boldsymbol{B}+\mathbf{1}) \geq 0.1$.

(c) $\# \boldsymbol{B} \in Z^{+}$.

Procedure for computation of $(u / c)$ :

S1 First is computed $\Delta H_{P}(\boldsymbol{B}+\mathbf{1}) \quad$ (resp. $\left.\Delta H_{D}(\boldsymbol{B}+\mathbf{1})\right)$.

S2 Fulfilling condition $(u / c) \in\langle 0,1\rangle$ (starting with an initial quantity of $(u / c))$ is calculated the quantity \#B.

S3 In the list of Ramsey numbers [15] we find numbers $R(3, \# \boldsymbol{B}), R(4, \# \boldsymbol{B}), \ldots, R(r 1, \# \boldsymbol{B})$.

S4 We find the nearest numbers $R(3, \# \boldsymbol{B}+\mathbf{1})$, $R(4, \# \boldsymbol{B}+\mathbf{1}), \ldots, R(r 2, \# \boldsymbol{B}+\mathbf{1})$.

S5 We compute combinations of Ramsey numbers for the selection of a minimal difference

$$
\Delta f(R N)=R N(x, \# \boldsymbol{B}+\mathbf{1})-R N(y, \# \boldsymbol{B}) .
$$

So we find numbers $R N(y, \# \boldsymbol{B})$ : $(p 1, p 2, p 3, p 4, p 5, \ldots), \quad$ and $\quad R N(x, \# \boldsymbol{B}+\mathbf{1})$ : $(q 1, q 2, q 3, q 4, q 5, \ldots)$, and we express the sequence $((q 1-p 1),(q 1-p 2),(q 2-p 2),(q 2-$ $p 3),(q 3-p 3), \ldots)$

S6 From this sequence is computed

$$
\Delta_{1} f(R N)=\min \{(q 1-p 1),(q 1-p 2),(q 2-p 2), \ldots\}
$$

S7 We turn back to step S2 and we select another $(u / c)$ (fulfilling condition (a)).

S8 We continue till the step S6 and compute

$$
\Delta_{2} f(R N)=\min \{\ldots\}
$$

S9 This procedure from S7 to S8 is repeated as many times as possible until the variability of the sequence $\left(\Delta_{1} f(R N), \ldots, \Delta_{k} f(R N)\right)$ is seen. 
S10 The best value of the quotient $(u / c)$ is a number for which we found

$$
\min \left\{\Delta_{1} f(R N), \ldots, \Delta_{k} f(R N)\right\}
$$

S11 For this value of $(u / c)$ we solve equation (7) (from subsection 3.2) and we continue in the method.

Note: In calculation in Section 4 of the paper the initial quantity of $(u / c)$ for S2 has been 0.35 . 